\title{
CA J oint Resource Allocation Algorithm Based on QoE Weight
}

\author{
LIU Jun-Xia ${ }^{1,2}$, JIA Zhen-Hong ${ }^{1}$ \\ ${ }^{1}$ School of Information Science and Engineering, Xinjiang University \\ Urumqi 830046, Xinjiang-P.R. China \\ [e-mail: 251414185@qq.com; jzhh@xju.edu.cn ] \\ ${ }^{2}$ Department of Electrical and Information, Xinjiang Institute of Engineering \\ Urumqi 830023, Xinjiang-P.R. China \\ *Corresponding author: JIA Zhen-Hong
}

Received May 10, 2017; revised November 5, 2017; accepted December 10, 2017; published May 31, 2018

\begin{abstract}
For the problem of cross-layer joint resource allocation (JRA) in the Long-Term Evolution (LTE)-Advanced standard using carrier aggregation (CA) technology, it is difficult to obtain the optimal resource allocation scheme. This paper proposes a joint resource allocation algorithm based on the weights of user's average quality of experience (JRA-WQOE). In contrast to prevalent algorithms, the proposed method can satisfy the carrier aggregation abilities of different users and consider user fairness. An optimization model is established by considering the user quality of experience (QoE) with the aim of maximizing the total user rate. In this model, user QoE is quantified by the mean opinion score (MOS) model, where the average MOS value of users is defined as the weight factor of the optimization model. The JRA-WQOE algorithm consists of the iteration of two algorithms, a component carrier (CC) and resource block $(\mathrm{RB})$ allocation algorithm called DABC-CCRBA and a subgradient power allocation algorithm called SPA. The former is used to dynamically allocate CC and RB for users with different carrier aggregation capacities, and the latter, which is based on the Lagrangian dual method, is used to optimize the power allocation process. Simulation results showed that the proposed JRA-WQOE algorithm has low computational complexity and fast convergence. Compared with existing algorithms, it affords obvious advantages such as improving the average throughput and fairness to users. With varying numbers of users and signal-to-noise ratios (SNRs), the proposed algorithm achieved higher average QoE values than prevalent algorithms.
\end{abstract}

Keywords: LTE-Advanced, carrier aggregation, resource allocation, quality of experience, discrete artificial bee colony algorithm

This research is supported by Natural Science Foundation of Xinjiang Uygur Autonomous Region of China (2017D01A20) 


\section{Introduction}

$\mathbf{W}_{\text {ith the continual evolution of communication technology, increasing number of mobile }}$ users, and growing user demands, spectral resources have become increasingly scarce. To achieve higher data transmission rates and better user quality of experience (QoE), carrier aggregation (CA) technology has been proposed in the 3GPP LTE-Advanced mobile communication standard. CA technology increases the system bandwidth by aggregating multiple continuous or discrete frequency bands to realize faster data transmission rates and higher spectrum utilization [1]. However, in practice, allocating component carrier (CC) and resource blocks (RBs) and assigning transmission power to users have lately emerged as pressing issues.

According to research on CA resource allocation in recent years, CC allocation methods can be divided into those based on round-robin scheduling and those based on free-hash scheduling $[1,2,3]$. Assuming that CC allocation has been determined, two schemes are available for allocating RBs: joint resource allocation scheme (JRS) and independent resource allocation scheme (IRS) [2,4,5]. In JRS, all CCs are combined into a "large carrier" for allocating RBs. JRS requires that each user continuously receive signals from all CCs simultaneously; this increases the complexity of signal processing and the power consumption of the user terminal $[6,7,8]$. In turn, this increases the complexity of resource allocation [9]. However, JRS can obtain the maximal frequency diversity gain and automatically balance loads among CCs; this is called the optimal resource allocation scheme in the LTE-Advanced CA system. In [2], a proportion-fair scheduler for cross-carriers was studied, and it was proved that the cross-carrier JRS is superior to IRS. In [3], an improved CC selection method was proposed for discontinuous carrier aggregation. In [8], for the cross-carrier scheduling problem in LTE-Advanced, a mathematical model based on logarithmic benefit was established. When the logarithm of the throughputs of all users is considered the optimization objective, optimal network benefit can be obtained. A cross-carrier resource scheduling algorithm was proposed in [10]. In [11], CCs and RBs were jointly considered and a suboptimal allocation algorithm was proposed. To reduce the complexity of resource allocation, CA resource allocation in IRS is divided into two steps: CC selection and RB allocation. In this scheme, a user can only use the RB on the selected CC for data transmission. Therefore, the scheme can only obtain low-frequency diversity gain, and the load imbalance among the CCs affects the system performance [2,12]; this results in poor performance of IRS, as described in [13] and [14]. [15] proposes a low-complexity and low-feedback-rate channel allocation approach for CA MIMO systems as an application for heterogeneous networks (HetNets) with multiple CCs and users.

The above studies did not consider the transmission power of each RB. In [16], a novel CA scheme was proposed for licensed/unlicensed MIMO LTE systems that optimally allocate the resources (power and RBs) of an eNodeB to UE (user equipment). Furthermore, the proposed approach handles coexisting matters in unlicensed bands in an efficient and decentralized manner. [17] studied the resource allocation problem for CA-enabled HetNets in which the spectrum owned by a macro-cell operator (MCO) can be shared by both unlicensed users (UUs) and licensed users (LUs). The authors also formulated a novel hierarchical game theoretic framework to jointly optimize the transmit powers and subband allocations of the UUs and the pricing strategies of the MCO. In [18], a resource scheduler was proposed by considering the CC, RB, and power allocation. However, CC and RB allocations were addressed separately from the issue of power allocation and were based on the suboptimal allocation method proposed in [11]. Therefore, the algorithm in [18] cannot yield optimal 
results. The problems of CC allocation, RB allocation, and power allocation were considered in [19] as well; however, the computational complexity of the proposed solution was high. In $[18,19]$, the problem of QoE was not considered. [20,21,22] focused on improving the quality-of-service (QoS) performance of CA systems. [20] guaranteed users' QoS requirements by satisfying the minimum transmission rate. A resource scheduling algorithm based proportional fairness (PF) approach is used to allocate CC, RB, and Modulation and Coding Scheme (MCS). [21] uses a utility PF approach. In this approach, users are partitioned into different groups based on the carrier coverage area. In each user group, RBs from all in-band carriers are assigned to UEs. In the case of average power allocation, each user is guaranteed a minimum QoS because no user is allocated zero RBs. [22] proposed joint user scheduling (JUS) based on Earliest Deadline First (EDF) for CC and RB allocation. However, $[20,21,22]$ did not consider power allocation and users' QoE.

To ensure user satisfaction, operators have shifted the focus of their services from QoS to QoE. As far as I know, there is only little literature on resource allocation considering user's QoE, especially in the CA technology scenario. [23] proposes a downlink cross-layer QoE-aware sub-carrier and power allocation algorithm in Heterogeneous OFDMA system. [24] researches the multi-channel energy-efficiency resource allocation of user's QoE in HetNet. [25] proposes an algorithm based device-to-device communication to enhance user's QoE in software defined multi-layer LTE-Advanced network. Therefore, a resource allocation scheduler that jointly considers the QoE of the CC, RBs, and power and low-computational complexity needs to be further studied for CA systems.

The rest of this paper is organized as follows. Chapter 2 describes the resource allocation problem based on QoE. Chapter 3 introduces the resource allocation algorithm, including discrete artificial bee colony algorithm; the proposed CC and RB allocation algorithm; the proposed power algorithm; and the proposed joint CC, RB, and power source allocation algorithms. Chapter 4 presents an analysis of the simulation results. Finally, Chapter 5 summarizes this study.

\section{Resource Allocation Problem Based on QoE}

The initial goal of network optimization is to improve the network QoS. However, QoS as an objective evaluation index of communication network performance depends on factors such as the bit error rate, packet loss rate, jitter, delay, etc. These factors depend on the communication network technology or transmission performance. Therefore, QoS cannot directly reflect a user's true attitude toward a business. According to the International Telecommunications Union (ITU), a user's QoE reflects the degree of subjective acceptance of use of the entire application or service, including the user's satisfaction with the usability, stability, integrity, and cost performance of the service [26,27,28]. QoE involves various factors, including not only objective factors related to network transmission but also subjective factors related to the user's experience. Therefore, QoE includes QoS.

LTE-Advanced is a further evolution based on LTE. It imposes more stringent requirements on system performance and customer service satisfaction. Only considering QoS as a service standard in LTE-Advanced cannot correctly reflect users' satisfaction. QoE is a direct reflection of user satisfaction that can not only reflect the impact of various QoS indices on user experience but also evaluate users' subjective experience of different businesses. Therefore, this paper focuses on the down-link resource allocation scheme by considering user QoE in LTE-Advanced CA technology. 


\subsection{QoE Quantification Model}

The mean opinion score (MOS) proposed by the ITU is widely used for QoE quantification and evaluation [28]. In this technique, QoE is divided into five grades, for which the corresponding MOSs are 5, 4, 3, 2, and 1 . Score of " 1 " and " 5 " represent "extremely low quality" and "excellent quality," respectively. The MOS quantification method reflects a user's subjective QoE. By using this quantification method, a user's subjective perception is mapped to an objective technical index. Different MOS quantification models are available for various services. Three types of businesses are considered in this paper: audio, video, and Web browsing. Different businesses can use pointed schedulers to further improve user satisfaction and resource utilization [29].

(1) Audio application

$M O S_{\text {audio }}$ is the MOS of audio businesses, and it is expressed as follows [23,30]:

$$
M_{\text {audio }}=a \log \left(b \overline{R(S)}\left(1-e_{m}\right)\right)
$$

where $\overline{R(S)}$ (bit/s) is the transmission rate of the audio user and $e_{m}$ is the target packet-error probability ( $e_{m}$ is directly related to the target bit error rate). The constants $a$ and $b$ are calculated when the transmission rate is known and there is no packet error $\left(e_{m}=0\right)$.

(2) Video application

$M O S_{\text {video }}\left(P S N R_{V}\right)$ is the MOS of video application businesses, and it is given by (2). This scoring model only applies to H.264/MPEG-4 AVC standard video services [23,30]:

$$
\operatorname{MOS}_{\text {video }}\left(P S N R_{V}\right)=\left\{\begin{array}{c}
1.0, P S N R_{V} \leq P S N R_{1.0} \\
d \log \left(P S N R_{V}\right), P S N R_{1.0}<P S N R_{V}<P S N R_{4.5} \\
4.5, P S N R_{V} \geq P S N R_{4.5}
\end{array}\right.
$$

where $P S N R_{V}$ represents the peak signal-to-noise ratio (PSNR) of the video service. As given by (3), the $P S N R_{V}$ value is related to the data rate of the video stream. $P S N R_{1.0}$ and $P S N R_{4.5}$ represent the PSNR corresponding to the user perceiving quality as being unacceptable and the user being "very satisfied" with the quality, respectively. The constant $d$ is determined by the PSNR, where the MOS value is in the interval [1.0,4.5]:

$$
P S N R_{V}=\mathrm{u}+\mathrm{v} \sqrt{\frac{\overline{R(S)}}{w}}\left(1-\frac{w}{\overline{R(S)}}\right)
$$

where $\overline{R(S)}$ is the transmission rate of the video user, and $\mathrm{u}, \mathrm{v}$, and $w$ are time-variable constants that can be obtained by matching the distortion model of the measured video bit stream.

(3) Web browsing application

The MOS of the Web browsing service is expressed as

$$
M O S_{w e b}=-k_{1} \ln (d(\overline{R(S)}))+k_{2}
$$

where $k_{1}(=1.1120)$ and $k_{2}(=4.647)$ are obtained by analyzing experimental results for a Web browsing application, $\overline{R(S)}$ is the transmission rate achieved by a Web browsing user, and $d(\overline{R(S)}$ is the time delay between the transmission and acceptance of a Web browsing sending request. The time delay depends on multiple factors such as the webpage size, protocol type, and round-trip time [31]. 


\subsection{CA Resource Allocation Optimization Model Based on QoE Weight}

This study addresses the joint resource allocation problem of CC, RB, and transmission power. It is assumed that a base station eNB (eNodeB) provides communication services for $\mathrm{K}$ users over N CCs in an LTE-A system. Different users can support different bandwidths. Broadband users can support more CCs, whereas narrow-band users can support fewer CCs, or even only one CC. $C_{k}$ indicates that user $k$ can support $C$ number of CCs; $C=$ $\left[C_{1}, C_{2}, \ldots, C_{k}\right]$ represents the numbers of CCs that different users can support, $n \in\{1,2, \ldots, N\}$ represents a group of CCs, and $k \in\{1,2, \ldots, K\}$ represents an end user, where one CC contains M RBs.

When the m-th RB of the n-th CC is allocated to user $k$, user $k$ can acquire rate $r_{n, m}^{k}(t)$ in time slot $t$. The rate is expressed as

$$
r_{n, m}^{k}(t)=B_{R B} \log _{2}\left(1+p_{n, m}^{k}(t) \gamma_{n, m}^{k}(t)\right)
$$

where $p_{n, m}^{k}(t)$ is the transmission power when the m-th RB of the n-th CC in time slot $t$ is allocated to user $k, B_{R B}$ is the bandwidth of an $\mathrm{RB}$, and $\gamma_{n, m}^{k}(t)$ is the channel state information when the m-th RB of the $\mathrm{n}$-th CC in time slot $t$ is allocated to user $k$ (assuming that the base station contains all channel state information). $\gamma_{n, m}^{k}(t)$ is expressed as

$$
\gamma_{n, m}^{k}(t)=\left|h_{n, m}^{k}(t)\right|^{2} / N_{0} B_{R B}
$$

where $h_{n, m}^{k}(t)$ denotes the channel gain, $N_{0}$ is the unilateral power spectral density of additive white Gaussian noise.

$\eta_{n, m}^{k}(t)$ denotes the assignment identifier of the RB. When the m-th RB of the n-th CC is allocated to user $k, \eta_{n, m}^{k}(t)=1$; otherwise, $\eta_{n, m}^{k}(t)=0 . \eta_{n, m}^{k}(t)$ needs to satisfy the constraint in (7):

$$
\sum_{k=1}^{K} \eta_{n, m}^{k}(t) \leq 1, \forall n, \forall m
$$

$\chi_{n}^{k}(t)$ represents the assignment identifier of the CC. When the n-th CC is allocated to user $k, \chi_{n}^{k}(t)=1$; otherwise, $\chi_{n}^{k}(t)=0 . \chi_{n}^{k}(t)$ needs to satisfy the constraint in (8):

$$
\sum_{n=1}^{N} \chi_{n}^{k}(t) \leq C_{k}, \forall k
$$

$P_{T}$ denotes the total transmission power. The base station allocates the total transmission power to each RB. Therefore, the actual power consumption of the system is the sum of powers on all RBs. Power $p_{n, m}^{k}(t)$ must satisfy the following constraint:

$$
\sum_{n=1}^{N} \sum_{m=1}^{M} \sum_{k=1}^{K} \chi_{n}^{k}(t) \eta_{n, m}^{k}(t) p_{n, m}^{k}(t) \leq P_{T}
$$

Let $R_{k}(t)$ denote the total rate obtained by user $k$ in time slot $t$, as expressed in (10):

$$
R_{k}(t)=\sum_{n=1}^{N} \sum_{m=1}^{M} \chi_{n}^{k}(t) \eta_{n, m}^{k}(t) r_{n, m}^{k}(t)
$$

Considering user $k$ 's average QoE as the weight factor, it can be defined as in (11):

$$
w_{k}(t)=\operatorname{mos}_{k}(n, t) / \sum_{k=1}^{K} \operatorname{mos}_{k}(n, t)
$$

where $\operatorname{mos}_{k}(n, t)$ is the instantaneous QoE score for user $k$ in time slot $t$, and it is calculated using the MOS quantification model described in Section 2.1, where the user rate is substituted into the instantaneous rate. 
In considering the user fairness condition, the optimal mathematical model that considers the user's QoE and maximizes the system's total rate is as follows (time slot variable $t$ is omitted for simplicity):

$$
\begin{aligned}
& P_{1}: \max _{p_{n, m}^{k}, \chi_{n}^{k}, \eta_{n, m}^{k}} \sum_{n=1}^{N} \sum_{m=1}^{M} \sum_{k=1}^{K} w_{k} \frac{1}{R_{k}(t)} B_{R B} \chi_{n}^{k} \eta_{n, m}^{k} \log _{2}\left(1+p_{n, m}^{k} \gamma_{n, m}^{k}\right) \\
& \text { s.t. (7): } \sum_{k=1}^{K} \eta_{n, m}^{k} \leq 1, \forall n, \forall m \\
& \text { (8): } \sum_{n=1}^{N} \chi_{n}^{k} \leq C_{k}, \forall k \\
& \text { (9): } \sum_{n=1}^{N} \sum_{m=1}^{M} \sum_{k=1}^{K} \chi_{n}^{k} \eta_{n, m}^{k} p_{n, m}^{k} \leq P_{T} \\
& \text { (13): } \chi_{n}^{k} \in\{0,1\}, \eta_{n, m}^{k} \in\{0,1\}, p_{n, m}^{k} \geq 0, \forall n, \forall m, \forall k
\end{aligned}
$$

where model $\mathrm{P}_{1}$ is defined as a total rate maximization problem based on the weighted average QoE, $\overline{R_{k}(t)}$ is the average rate of user $k$ from time 0 to $t$. The time-window smoothing technique is employed, as in (14):

$$
\overline{R_{k}(t)}=\left(1-\frac{1}{t_{C}}\right) \overline{R_{k}(t-1)}+\frac{1}{t_{C}} R_{k}(t)
$$

where $t_{C}$ represents the length of the smoothing time window. Condition (7) indicates that an $\mathrm{RB}$ can only be allocated to one user in the same time slot. Condition (8) implies that the number of CCs allocated to users must satisfy the constraints of each user by supporting the maximum number of CCs because each user has different CA capabilities. Condition (9) indicates that the sum of the consumed power at each allocated RB should be less than or equal to the total transmission power. Condition (13) indicates that the CC assignment identifier and $\mathrm{RB}$ assignment identifier are 0 and 1 , respectively, and the power assigned to each RB is nonnegative.

The physical meaning of the optimization model $\mathrm{P}_{1}$ is the maximization of the total user rate, where the average user QoE is taken as the weight factor for considering user fairness and the satisfaction of CA capabilities under different users' conditions.

\section{Resource allocation algorithm}

$\mathrm{P}_{1}$ is a nonlinear mixed optimization problem that is divided into two subproblems: the CC and $\mathrm{RB}$ allocation problem and the power allocation problem. The former is addressed when power allocation has been determined. Once the CC and RB allocations are complete, power is allocated to further improve the overall system performance. In this paper, a discrete artificial bee colony optimization algorithm (DABC) is used for $\mathrm{CC}$ and $\mathrm{RB}$ allocation. The basic principles of DABC are introduced below.

\subsection{DABC Algorithm}

The ABC algorithm is inspired by the food foraging behavior of honey bees. Honey bees are divided into three groups: employed bees, onlooker bees, and scout bees. These bees 
complete different tasks at each stage according to the division of labor. They search for the optimal solution to a problem by collecting and sharing information concerning food sources. The employed bees and onlooker bees exploit the food source, namely, nectar. The scout bees observe whether the search falls into a local optimal solution. Every food source represents a possible solution, and fitness is determined in terms of the quality of the food source. In the search process, SN initial solutions $\theta_{i}(t)(i=1,2, \ldots, S N)$ are generated, where $S N$ is the number of food sources. Every solution is a d-dimensional vector. In the t-th search, the location of the i-th honey source is given by

$$
\theta_{i}(t)=\left\{\theta_{i, 1}(t), \theta_{i, 2}(t), \ldots, \theta_{i, d}(t)\right\} \in R^{d}
$$

Following initialization, all honey bees search in circles until the iterator reaches the maximum number of iterations or satisfies the given minimal error [32,33].

At the beginning of the search stage, every employed bee updates the position of the food source by

$$
\theta_{i, j}(t+1)=\theta_{i, j}(t)+\phi_{i, j}\left(\theta_{i, j}(t)-\theta_{k, j}(t)\right)
$$

where $\phi_{i, j}$ is the weight of the length of the random search step in the range [-1,1], $k \in$ $\{1,2, \ldots, S N\}, j \in\{1,2, \ldots, d\}$, and $k \neq i$. In comparing the fitness of the old food source with that of the new one, if the latter is better than the former, it replaces the old one; otherwise, the old food source is retained and the new one is discarded. Once all employed bees have completed one search iteration, the employed bees share food source information through a waggle dance. The probability of each food source is calculated by the onlookers as in (17):

$$
P_{i}=\frac{H\left(\theta_{i}(t)\right)}{\sum_{k=1}^{M} H\left(\theta_{k}(t)\right)}
$$

A random number within the range $[0,1]$ is then generated. If the probability of the solution is higher than the random number, the onlooker bees generate a new solution using (16). In comparing the fitness values of the old and new food sources, if the fitness value of the new food source is better than that of the old one, it replaces the old one; otherwise, the old solution is retained. Once all honey bees have completed one search iteration, if the solution cannot be further improved by limit iterations, the food source is discarded, and the corresponding honey bee becomes a scout bee. This scout bee searches for a new food source randomly. Moreover, $\theta_{\max }$ is the global optimal solution. After every iteration, if $H\left(\theta_{i}(t+1)\right)>$ $H\left(\theta_{i}(t)\right), \theta_{\max }$ is updated, that is, $\theta_{\max }=\theta_{i}(t+1)[34,35,36]$.

As the classic artificial bee colony algorithm is suitable for the continuous domain optimization problem, it cannot be directly used for discrete variable in the CC and RB optimization problem. To implement the artificial bee colony algorithm in the discrete domain, the sigmoid function discrete method is used to map the continuous domain of the ABC algorithm into a binary discrete domain; the mapping formula is $[37,38]$

$$
\theta_{i, j}(t+1)=\left\{\begin{array}{c}
1, \operatorname{rand}(1)<\frac{1}{1+\exp \left(-\theta_{i, j}(t+1)\right)} \\
0, \text { others }
\end{array}\right.
$$

\subsection{CC and RB Allocation Algorithm Based on DABC}

If the power allocation is known, the problem of the joint assignment of CC and RB can be expressed as $P_{11}$ : 


$$
\begin{gathered}
P_{11}: \max _{\chi_{n}^{k}, \eta_{n, m}^{k}} \sum_{n=1}^{N} \sum_{m=1}^{M} \sum_{k=1}^{K} w_{k} \frac{1}{R_{k}(t)} B_{R B} \chi_{n}^{k} \eta_{n, m}^{k} \log _{2}\left(1+p_{n, m}^{k} \gamma_{n, m}^{k}\right) \\
\text { s.t. (7): } \sum_{k=1}^{K} \eta_{n, m}^{k} \leq 1, \forall n, \forall m \\
\text { (8): } \sum_{n=1}^{N} \chi_{n}^{k} \leq C_{k}, \forall k \\
(20): \chi_{n}^{k} \in\{0,1\}, \eta_{n, m}^{k} \in\{0,1\}, \forall n, \forall m, \forall k
\end{gathered}
$$

As $P_{11}$ is an NP-hard problem, we can use an artificial intelligence algorithm to obtain the optimal CC and RB allocation scheme. follows:

When the power allocation is known, the proof that $P_{11}$ is an NP-hard problem is as

\section{Proof:}

First, it is proved that the joint assignment problem $P_{11}$ of CC and RB can be mapped to a 0-1 multidimensional backpack problem.

(1) 0-1 multidimensional knapsack problem (0-1MKP): 0-1MKP comprises $m$ items and $n$ knapsacks with different capacities $C a_{i}$, where $i \in\{1,2, \ldots, n\}$ and $n$ is the number of knapsacks. Each item $j$ has profit $p_{j}$, where $j \in\{1,2, \ldots, m\}$ and $m$ is the number of items. Item $j$ is placed in knapsack $i$; this will generate the weight $\mathrm{w}_{\mathrm{ij}}$. $\mathrm{x}_{\mathrm{j}}$ is the assignment index that indicates whether item $j$ is placed in the knapsack; $x_{j}=1$ indicates that item $\mathrm{j}$ is placed in the knapsack, and $x_{j}=0$ indicates that item $j$ is not placed in the knapsack. The goal of $0-1 \mathrm{MKP}$ is to maximize the profit generated by placing the item in the knapsack without exceeding the capacity of each backpack. The mathematical expression of 0-1MKP is [39]:

$$
\begin{gathered}
\max \sum_{j=1}^{m} p_{j} \mathrm{x}_{\mathrm{j}} \\
\text { s.t. }(21-1) \sum_{j-1}^{m} \mathrm{w}_{\mathrm{ij}} \mathrm{x}_{\mathrm{j}} \leq C a_{i}, \forall i \\
(21-2) \mathrm{x}_{\mathrm{j}} \in\{0,1\}, \forall j
\end{gathered}
$$

(2)Mapping of $P_{11}$ to $0-1 \mathrm{MKP}$ : Assume that the power allocation is known and that each user can only use one RB. K users represents the K knapsacks of 0-1MKP. The CA capability $C_{k}$ of each user represents the capacity of each knapsack. RB represents the items; there are $N \times M$ items, where $\mathrm{N}$ is the number of CCs and $\mathrm{M}$ is the number of RBs in each CC. An RB (item) is assigned to (placed in) a user (knapsack) that will generate weight "1." An RB in a CC is assigned to (placed in) a user (knapsack) to achieve a rate (generate profit) $r_{n, m}^{k} \cdot \chi_{n}^{k} \eta_{n, m}^{k}$ represents whether an RB is assigned to a user (an item is placed in the backpack). The objective function and constraints of $P_{11}$ are mapped to maximize the total profits of $0-1 \mathrm{MKP}$ when the knapsack capacity is not exceeded.

At this point, the $\mathrm{CC}$ and $\mathrm{RB}$ joint distribution problem $P_{11}$ is mapped to a multidimensional 0-1 knapsack problem.

Second, as the multidimensional 0-1 knapsack problem is a well-known NP-hard 
problem, $P_{11}$ is also an NP-hard problem. problem.

Finally, it is proved that the joint assignment problem $P_{11}$ of CC and RB is an NP-hard

\section{End of proof.}

The discrete optimization problem $P_{11}$ is an NP-hard problem. The DABC optimization theory is used to solve $P_{11}$. The designed objective function of the DABC algorithm is expressed as

$$
\begin{gathered}
\mathrm{f}(\mathrm{t})=\mathrm{H}\left(\theta_{i}(t)\right)=\sum_{n=1}^{N} \sum_{m=1}^{M} \sum_{k=1}^{K} w_{k} \frac{1}{R_{k}(t)} B_{R B} \chi_{n}^{k} \eta_{n, m}^{k} \log _{2}\left(1+p_{n, m}^{k} \gamma_{n, m}^{k}\right) \\
\text { s.t. (7): } \sum_{k=1}^{K} \eta_{n, m}^{k} \leq 1, \forall n, \forall m \\
\text { (8): } \sum_{n=1}^{N} \chi_{n}^{k} \leq C_{k}, \forall k \\
(20): \chi_{n}^{k} \in\{0,1\}, \eta_{n, m}^{k} \in\{0,1\}, \forall n, \forall m, \forall k
\end{gathered}
$$

\subsubsection{Definition of Nectar Position}

The position of the source of honey is an M-dimensional vector as follows:

$$
\theta_{i}=\left\{\theta_{i, 1}, \theta_{i, 2}, \ldots, \theta_{i, M}\right\}, i=1,2, \ldots, N
$$

where $N$ is the number of CCs and $M$ is the number of RBs in one CC. Then, the allocation scheme matrix of the positions of the honey source is $F_{a}$, and it is expressed as

$$
F_{a}=\left[\begin{array}{ccc}
\theta_{1,1} & \cdots & \theta_{1, M} \\
\vdots & \ddots & \vdots \\
\theta_{N, 1} & \cdots & \theta_{N, M}
\end{array}\right]
$$

where the allocation scheme matrix element $\theta_{n, m}$ is a binary variable. $\theta_{n, m}=1$ indicates that the m-th RB of the n-th CC is allocated to the user, and $\theta_{n, m}=0$ implies that the m-th RB of the $n$-th CC is not occupied.

\subsubsection{Dealing with Constraint Violations}

$f c_{j}\left(\theta_{i}\right)$ is defined according to whether individual $\theta_{i}$ violates the $\mathrm{j}$-th constraint; the expression is as follows:

$$
f c_{j}\left(\theta_{i}\right)=\left\{\begin{array}{l}
1, \theta_{i} \text { violates the constraint condiction } \mathrm{j} \\
0, \theta_{\mathrm{i}} \text { satisfies the constraint condiction } \mathrm{j}
\end{array}\right.
$$

$\operatorname{Fc}\left(\theta_{i}\right)$ represents the degree to which individual $\theta_{i}$ violates all constraints; the expression is as follows:

where $p c$ is the number of constraints.

$$
F c\left(\theta_{i}\right)=\sum_{j}^{p c} f c_{j}\left(\theta_{i}\right) / p c
$$

According to the idea in [40], the maintenance of the ratio of non-feasible solutions with probability $p_{t}$ is used in the comparison strategy of the individual, which is as follows.

When $\theta_{1}$ and $\theta_{2}$ are feasible solutions, by comparing their objective functions, the individual with the larger value is dominant. When $\theta_{1}$ and $\theta_{2}$ are both unfeasible solutions, by comparing their degrees of violations of constraints, the individual with smaller $F c\left(\theta_{i}\right)$ is 
dominant. When $\theta_{1}$ is a feasible solution and $\theta_{2}$ is not, if $\operatorname{rand}()<p_{t}$, by comparing their objective functions, the larger value is dominant; otherwise, $\theta_{1}$ is dominant.

\subsubsection{DABC-CCRBA Algorithm}

According to the above definition and the improved method, the discrete artificial bee colony algorithm optimization problem $P_{11}$ optimizes the CC and RB allocation algorithm (DABC-CCRBA). The DABC-CCRBA algorithm involves the following steps:

(1) Parameter settings: number of honey sources SN, number of employed bees = number of onlooker bees $=S N$, number of iterations $i m i t$, maximum number of iterations $t_{\max }$, number of CCs $N=S N$, number of RBs on each CC $M=d$, probability $p_{t}$ of using the objective function value for comparison, and initial number of iterations $t=0$.

(2) Initialize the position of nectar sources and allocation scheme matrix $F_{a}$, calculate the fitness value of all nectar sources, and record the fitness value of the initial position as the initial optimal solution $\theta_{\text {max }}$.

(3) Employed bees' stage: The employed bees search for new solutions from the neighborhood according to (16) and (18). If the new nectar source is better than the given source, update the nectar source. Otherwise, discard the update.

(4) Onlooker bees' stage: According to the waggling dance of the employed bees, the onlooker bees have information concerning the fitness of the nectar sources. According to the probability obtained by (17), use the roulette method to select the food source. Then, the new food source is generated according to (16) and (18). At the same time, calculate the fitness of the new food source and compare it with the old one to determine whether the nectar source needs to be updated. Then, record the number of times the nectar source is not updated.

(5) Scout bees' stage: According to the recorded number of nectar sources that are not updated, determine whether sources are not updated more than limit times. If they are, the onlooker bees corresponding to the honey source become scout bees, and they randomly search for new nectar sources using (16) and (18).

(6) Compare the optimal solution of the given iteration with the global optimal solution of the last iteration, and choose the better one as the global optimal solution of the given iteration.

(7) Update iteration variable $t=t+1$, and judge whether it is greater than $t_{\max }$. If $t>t_{\max }$, the algorithm ends, and the resource allocation scheme $F_{a}$ corresponding to the optimal solution is recorded. Otherwise, return to Step (3).

The computational complexity of the DABC-CCRBA algorithm is $O(N M)$, compared with the computational complexities $O\left(N M K_{1}\right)$ of the MSUL algorithm (minimizing system utility loss)[18] and $O\left(N K_{1} K \sum_{i \in N} R B_{i} \sum_{k \in k_{1}} C_{k}\right)$ of the MSU algorithm (maximum system utility) ( $K_{1}$ is the number of narrow-band users[19]). The computational complexity of the proposed DABC-CCRBA algorithm is the lowest.

\subsection{SPA Power Allocation Algorithm}

When the CC and RB allocations are known, power allocation can be determined by problem $P_{12}$

$$
\begin{gathered}
P_{12}: \max _{p_{n, m}^{k}} \sum_{\substack{k=1 \\
k \in k(n, m)}}^{K} \sum_{n=1}^{N} \sum_{m=1}^{M} w_{k} \frac{1}{R_{k}(t)} B_{R B} \log _{2}\left(1+p_{n, m}^{k} \gamma_{n, m}^{k}\right) \\
\text { s.t. } \sum_{n=1}^{N} \sum_{m=1}^{M} \sum_{k=1}^{K} \chi_{n}^{k} \eta_{n, m}^{k} p_{n, m}^{k} \leq P_{T} \\
p_{n, m}^{k} \geq 0, \forall n, \forall m, \forall k
\end{gathered}
$$


where $k(n, m)$ denotes the user to which the m-th RB of the n-th CC is allocated. The objective function of the power optimization problem is expressed in (27). It shows that the objective function is a concave function and that the constraints are linear. Therefore, the power optimization problem $\mathrm{P}_{12}$ involves concave programming. The optimal solution is obtained using the Lagrangian dual method [41]. The Lagrangian function is defined as follows:

$$
\begin{aligned}
& L\left\{\left(p_{n, m}^{k}\right), \lambda\right\}=\sum_{\substack{k=1 \\
k \in k(n, m)}}^{K} \sum_{n=1}^{N} \sum_{m=1}^{M} w_{k} \frac{1}{R_{k}(t)} B_{R B} \log _{2}\left(1+p_{n, m}^{k} \gamma_{n, m}^{k}\right)+\lambda\left(P_{T}-\right. \\
& \left.\sum_{\substack{k=1 \\
k \in k(n, m)}}^{K} \sum_{n=1}^{N} \sum_{m=1}^{M} p_{n, m}^{k}\right)
\end{aligned}
$$

where $\lambda$ is the Lagrangian dual variable. The Lagrangian dual function of problem $P_{12}$ is defined as $g(\lambda)$, and it is expressed as

$$
g(\lambda)=\max _{p_{n, m}^{k}} L\left\{\left(p_{n, m}^{k}\right), \lambda\right\}
$$

Then, the dual programming of $P_{12}$ is as follows:

$$
\min _{\lambda>0} g(\lambda)=\min _{\lambda>0} \sum_{n, m} g_{n}(\lambda)+\lambda P_{T}
$$

where

$$
g_{n}(\lambda)=\max _{p_{n, m}^{k}} w_{k} \frac{1}{\overline{R_{k}(t)}} B_{R B} \log _{2}\left(1+p_{n, m}^{k} \gamma_{n, m}^{k}\right)-\lambda p_{n, m}^{k}
$$

The minimum value of $g_{n}(\lambda)$ is obtained by finding the partial derivative of $p_{n, m}^{k}$, namely,

$$
\frac{\partial g_{n}(\lambda)}{\partial p_{n, m}^{k}}=0 \Rightarrow p_{n, m}^{k}=\left[\frac{w_{k} B_{R B}}{\lambda \overline{R_{k}(t)}}-\frac{1}{\gamma_{n, m}^{k}}\right]^{+}, k \in k(n, m)
$$

where $[x]^{+}=\max (0,1)$. The subgradient searching is used to obtain the optimal power allocation scheme; all $\lambda$ are defined as $\lambda^{\prime}$ Then,

$$
g\left(\lambda^{\prime}\right) \geq g(\lambda)+d^{T}\left(\lambda^{\prime}-\lambda\right)
$$

where $d$ is the subgradient of $g(\lambda)$, and the subgradient of the Lagrangian dual function $g(\lambda)$ of problem $P_{12}$ is defined as

\section{Proof:}

$$
d=P_{T}-\sum_{n, m} p_{n, m}^{k *}, k \in k(n, m)
$$

$$
\begin{aligned}
& \because g\left(\lambda^{\prime}\right)=\max \sum_{n, m} w_{k} \frac{1}{\overline{R_{k}(t)}} B_{R B} \log _{2}\left(1+p_{n, m}^{k} \gamma_{n, m}^{k}\right)+\lambda^{\prime T}\left(P_{T}-\sum_{n, m} p_{n, m}^{k}\right) \\
& \therefore g\left(\lambda^{\prime}\right) \geq \sum_{n, m} w_{k} \frac{1}{\overline{R_{k}(t)}} B_{R B} \log _{2}\left(1+p_{n, m}^{k} \gamma_{n, m}^{k *}\right)+\lambda^{\prime T}\left(P_{T}-\sum_{n, m} p_{n, m}^{k *}\right)= \\
& \sum_{n, m} w_{k} \frac{1}{\overline{R_{k}(t)}} B_{R B} \log _{2}\left(1+p_{n, m}^{k} \gamma_{n, m}^{k *}\right)+\lambda^{T}\left(P_{T}-\sum_{n, m} p_{n, m}^{k *}\right)- \\
& \lambda^{T}\left(P_{T}-\sum_{n, m} p_{n, m}^{k *}\right)+\lambda^{\prime T}\left(P_{T}-\sum_{n, m} p_{n, m}^{k *}\right)=g(\lambda)+\left(P_{T}-\sum_{n, m} p_{n, m}^{k *}\right)^{T}\left(\lambda^{\prime}-\lambda\right) \\
& \text { where } p_{n, m}^{k *} \text { is the optimal power variable. }
\end{aligned}
$$

By comparing (35) and (33), we can obtain $g\left(\lambda^{\prime}\right) \geq g(\lambda)+d^{T}\left(\lambda^{\prime}-\lambda\right)$.

So the expression for the subgradient is obtained as given in (34).

\section{End of proof}

The update method for $\lambda$ in the optimal power expression (31) is the subgradient method in [41]. The update expression for $\lambda$ is given by

$$
\lambda^{(i+1)}=\left[\lambda^{(i)}+\frac{1}{i}\left(P_{T}-\sum_{n, m} p_{n, m}^{k}\right)\right]^{+}, k \in k(n, m)
$$

where $\frac{1}{i}$ is the update step scalar sequence of $\lambda$ in the subgradient direction, and $i$ is the number of iterations.

In this section, the power allocation algorithm for power allocation problem $P_{12}$ is defined as the subgradient power allocation algorithm (SPA). SPA involves the following 
steps:

(1) Input: CC and RB allocation scheme $F_{a}$; weight $w_{k}$ of each user QoE.

(2) Initialization: $p_{n, m}^{k}=P_{T} / M \times N, \gamma_{n, m}^{k}=\gamma_{n, m}^{k \in k(n, m)}, w_{k}=w_{k \in k(n, m)}, \forall n, \forall m, \lambda^{(0)} \geq 0$, number of iterations $i=0$.

(3) While $\left\|\lambda^{(i)}-\lambda^{(i-1)}\right\|<\varepsilon$, do

calculate the power allocation of each RB according to (32),

update $\lambda$ according to (36).

End while;

(4) Power allocation $\left\{p_{n, m}^{k}, \forall n, \forall m\right\}$ on each RB is the output.

\subsection{CA Joint Resource Allocation Algorithm Based on Average User QoE Weight}

In this paper, the optimization model of CA resource allocation is problem $\mathrm{P}_{1}$. In the context of considering fairness among users, the system total rate is maximized while considering user QoE. $\mathrm{P}_{1}$ is divided into two subproblems $\mathrm{P}_{11}$ and $\mathrm{P}_{12}$ that are iterated repeatedly until the $\mathrm{P}_{1}$ value of the optimization model ceases to increase. We define the CA resource allocation algorithm as a joint resource allocation algorithm based on the weights of the user's average QoE (JRA-WQOE), and its computational complexity is $O(N M+$ $\left.N M K / \varepsilon^{2}\right) . \varepsilon$ is the iteration termination condition. The JRA-WQOE algorithm has lower computational complexity than JCRPA and CARA. The computational complexity of JCRPA is $O\left(N M K_{1}+N M K / \varepsilon^{2}\right)$ [18] and that of CARA is $O\left(T_{\text {slot }} \chi N^{2} K^{3} M\right)$ [19].

The steps in the JRA-WQOE algorithm are as follows:

(1) Initialize the required parameters, and input the average QoE weight $\left\{w_{k}, \forall k\right\}$ for each user; each user can support the maximum number of CCs in set $\left\{C_{k}, \forall k\right\}$.

(2) $p_{n, m}^{k}=P_{T} / M \times N, \forall n, \forall m$.

(3) Call DABC-CCRBA, and dynamically gain the CC and RB allocation scheme.

(4) While the $P_{1}$ value does not increase, do

$t=t+1$;

call the SPA power allocation algorithm to allocate power to each RB;

call the DABC-CCRBA algorithm to allocate frequency resources CC and RB for the user.

End while;

(5) Output: CC and RB allocation scheme $F_{a}$, and power allocation on each RB $\left\{p_{n, m}^{k}, \forall n, \forall m, \forall k\right\}$.

\section{Simulation and Performance Analysis}

To further analyze the performance of the proposed JRA-WQOE algorithm, its average convergence, throughput, fairness, and average user QoE were analyzed through numerical simulations. In the LTE-A network, the downlink performance of CA technology was simulated. The cell radius was $1 \mathrm{~km}$. The base station eNB was at the center of the cell, and users (including narrow-band users and broadband users) were randomly distributed inside the cell. The main parameters of the simulation are shown in Table 1. 
Table 1. Main parameters of simulation test

\begin{tabular}{|c|c|}
\hline Parameters of the simulation & Parameter settings \\
\hline \hline Number of CCs; bandwidth of each CC & 5 CCs; bandwidth of each CC was $20 \mathrm{MHz}$ \\
\hline Each CCs contains the number of RBs & 100 \\
\hline Maximum total power $\mathrm{P}_{\mathrm{T}}$ & $45 \mathrm{dBm}$ \\
\hline Path loss & $P L(d)=137.74+5.22 \log (d)$ \\
\hline Thermal noise power spectral density & $-174 \mathrm{dBm} / \mathrm{Hz}$ \\
\hline Standard deviation of shadowing loss & $7 \mathrm{~dB}$ \\
\hline Small-scale fading distribution & Rayleigh fading \\
\hline
\end{tabular}

We have conducted 50 simulation experiments on the proposed JRA-WQOE algorithm, and these algorithms can all fast converge in each experiment. Fig. 1 shows the convergence of the JRA-WQOE algorithm when the number of users was 20. It can be seen that it can converge speedily. The JRA-WQOE algorithm only use six iterations to achieve convergence.

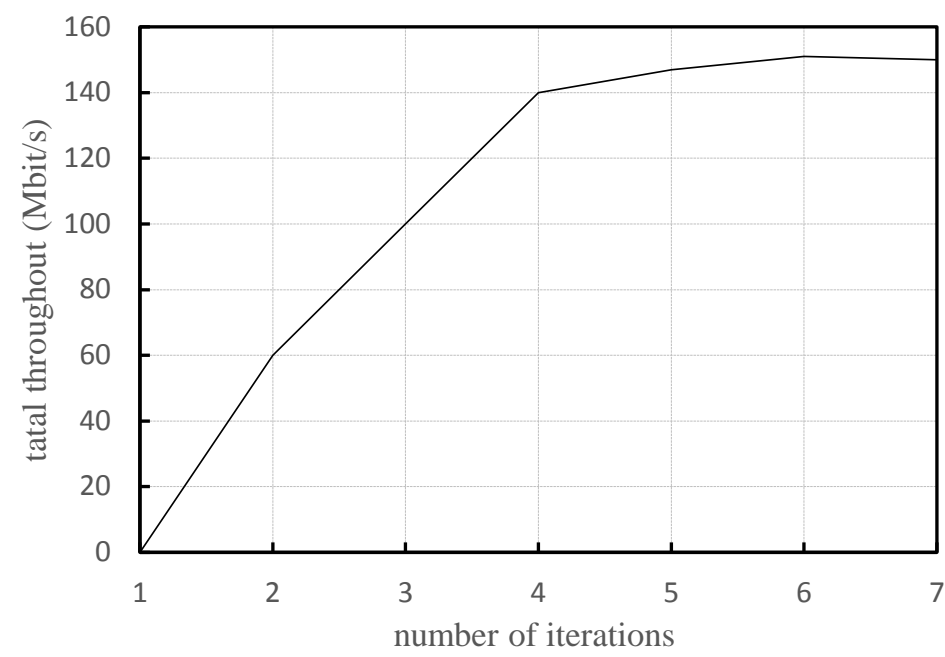

Fig. 1. Convergence of JRA-WQOE algorithm

The simulation experiments were designed to compare the performance of the following four resource allocation algorithms: proposed JRA-WQOE. DABC-CCRBA combined with equal power allocation algorithm (DABC-CCRBA + EPA), where the CC and RB allocation algorithm was based on DABC-CCRBA from Section 3.2. Joint CC, RB, and power resource allocation algorithm (JCRPA)[18]. And cross-CC-PF algorithm [2] combined with the equal power allocation algorithm (Cross-CC-PF + EPA), where the cross-CC-PF spectral resource allocation algorithm has been shown to be the optimal static carrier allocation algorithm.

The average throughputs of the four algorithms are shown in Fig. 2. The number of narrow-band users was equal to those of broadband users; numbers 1-10 on the abscissa represent narrow-band users and 11-20 represent broadband users. Compared with the other three algorithms, JRA-WQOE significantly improved average user throughput, especially the average throughput of broadband users. This is because the artificial intelligence algorithm was used to allocate $\mathrm{CC}$ and $\mathrm{RB}$ resources in the JRA-WQOE algorithm by considering user QoE. The DABC-CCRBA + EPA algorithm was less efficient than JRA-WQOE in improving the average throughput of users because the equal power allocation strategy was adopted in the 
power allocation process. The average throughputs of JRA-WQOE and DABC-CCRBA+ EPA were higher than those of the JCRPA algorithm and cross-CC-PF + EPA algorithm. The dynamic resources allocation of $\mathrm{CC}$ and $\mathrm{RB}$ and the adaptive power allocation method were adopted in the JCRPA algorithm. It achieved higher average throughput than the cross-CC-PF +EPA algorithm, as this is a static resource allocation algorithm.

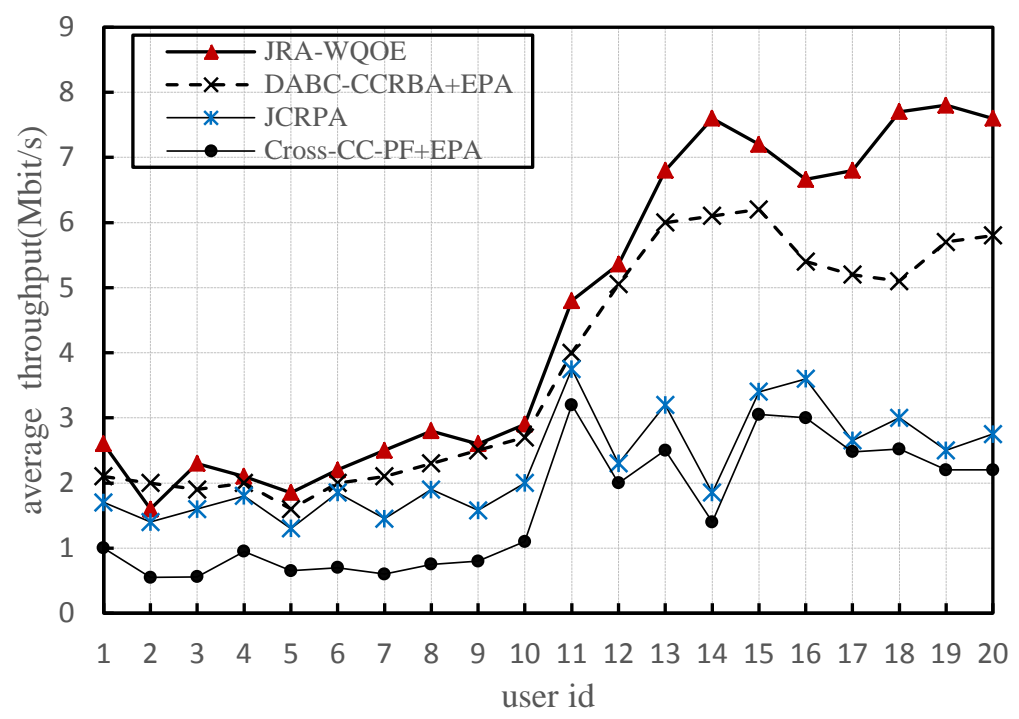

Fig. 2. Comparison of average user throughput

The fairness to users was measured using Jain's fairness index, which is expressed as

$$
F=\left(\sum \overline{R(k)}\right)^{2} / K \sum(\overline{R(k)})^{2}
$$

where $\overline{R(k)}$ represents the average transmission rate of user $k$, F value is [1/K, 1], and $\mathrm{K}$ is the number of users. When $F=1$, it means that all users had the same average transmission rate. The greater the $F$ value, the better is the fairness. The user fairness comparison is show in Fig. 3.

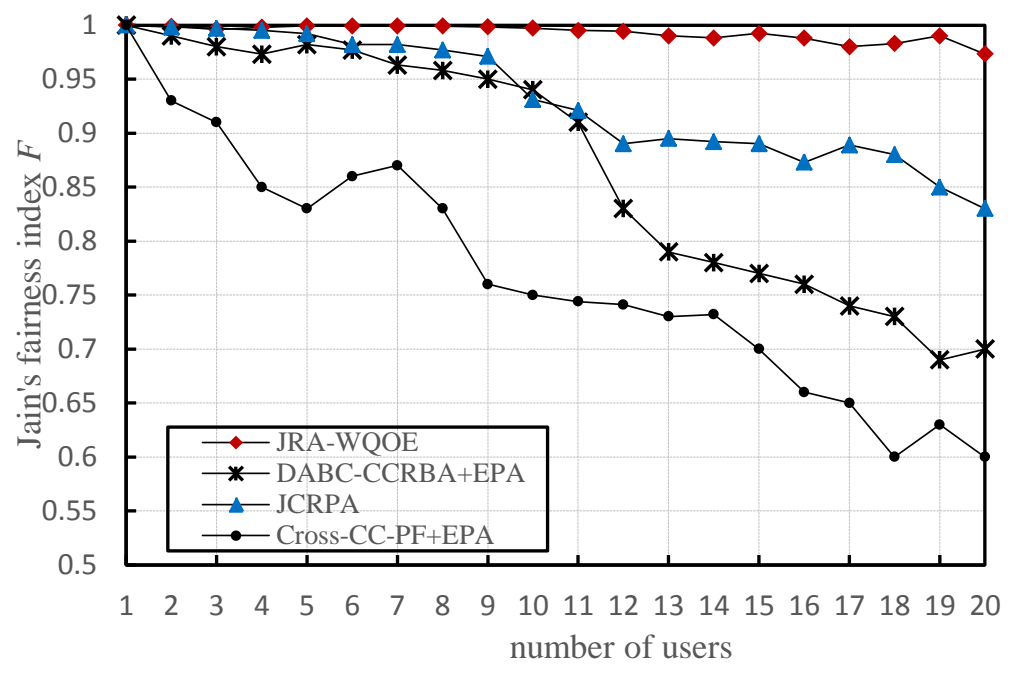

Fig. 3 Comparison of user fairness 
Fig. 3 shows that the JRA-WQOE algorithm had the best fairness. With an increase in the number of users, fairness decreased slightly primarily owing to user QoE. The fairness of JCRPA was better than that of DABC-CCRBA + EPA. The cross-CC-PF + EPA is a static resource allocation algorithm, and therefore, its fairness was the worst.

The average user QoE is expressed by (11). In the resource allocation model, the average user QoE was defined by the weight coefficient $w_{k}(t)$. We redefined the average user QoE as $Q o E_{\text {average }}$, and it is expressed as

$$
Q o E_{\text {average }}=w_{k}(t)=\operatorname{mos}_{k}(n, t) / \sum_{k=1}^{K} \operatorname{mos}_{k}(n, t)
$$

Fig. 4 shows simulation results of average user QoE that were compared in a communication network containing different numbers of users.

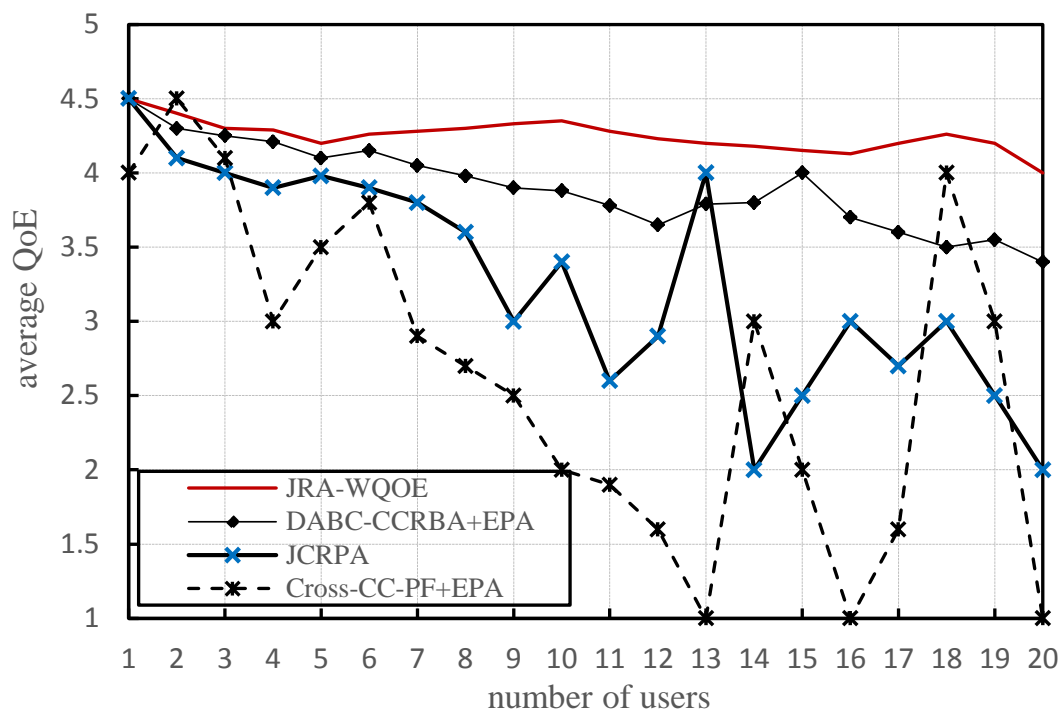

Fig. 4. Comparison of average user QoE based on different numbers of users

Fig. 4 shows that the JRA-WQOE algorithm improved the average user QoE. With an increase in the number of users, the system had higher $Q o E_{\text {average }}$ and the variation was stable. The DABC-CCRBA + EPA algorithm also had a satisfying average user QoE, and the change in the trend was relatively gentle. This is mainly because the above two algorithms considered user QoE. The power distribution of the DABC-CCRBA + EPA algorithm was the average allocation method, and the user $Q o E_{\text {average }}$ value of DABC + EPA was slightly lower on the whole than that of the JRA-WQOE algorithm. For the JCRPA algorithm and cross- PF + EPA, the user $Q o E_{\text {average }}$ values were lower than those of the JRA-WQOE algorithm and DABC-CCRBA + EPA. The average user QoE of cross-CC-PF + EPA was the worst, mainly because it is static frequency resource allocation combined with equal power allocation.

Fig. 5 shows that the average user QoE changed with SNR values when the number of users was 20. 


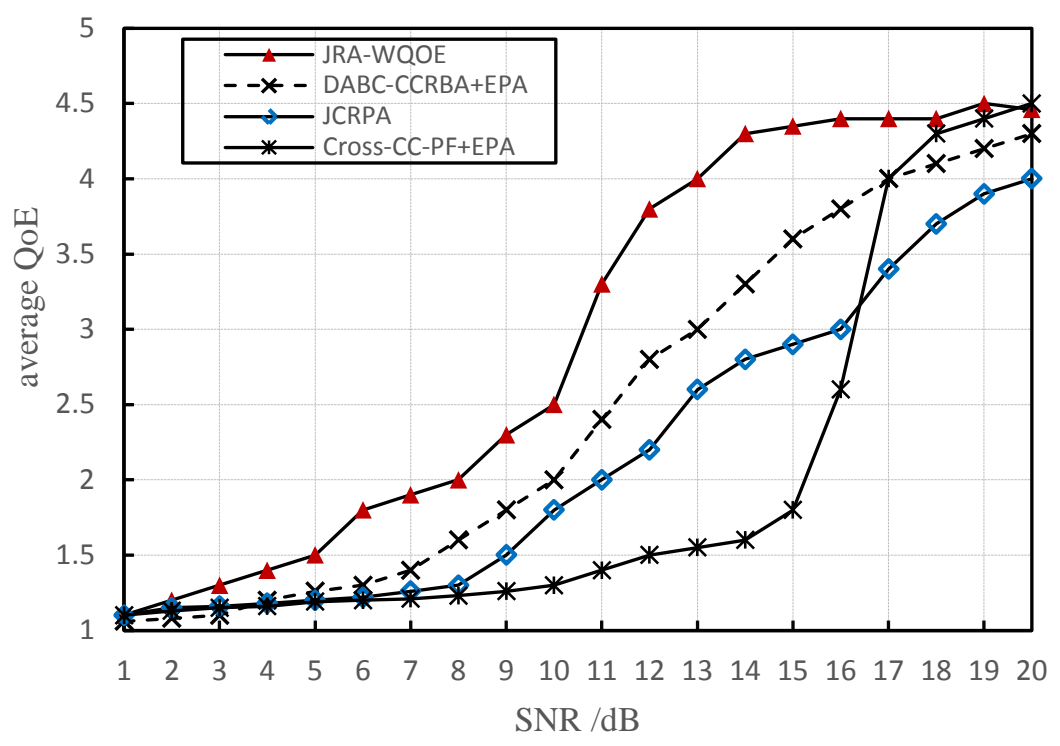

Fig. 5. Comparison of average user QoE for different SNRs

Fig. 5 shows that the average user QoE values increased with SNR. When $\mathrm{SNR} \leq 10$, the average QoE of each algorithm was not satisfied because the system resources could not meet all user needs. The average user QoE of the JRA-WQOE algorithm increased rapidly with SNR, and it was the maximum among the four algorithms. The average user QoE of DABC-CCRBA+EPA was the second highest, and those of JCRPA and cross-CC-PF + EPA algorithms were generally low. This is because they are designed to ignore user QoE, and therefore, they can only satisfy a part of it. Figs. $\mathbf{4}$ and $\mathbf{5}$ show that the proposed JRA-WQOE algorithm can improve the resource utilization rate from the perspective of user experience.

\section{Conclusion}

In this paper, the resource allocation problem in the context of newly added CA technology was studied in an application scenario in the LTE-Advanced standard. To further improve user satisfaction, communications operators have shifted focus from objective network QoS to subjective user QoE. Therefore, the QoE quantification MOS models of three application businesses were studied and analyzed. On this basis, the mathematical model $P_{1}$ was established, which is a joint resource allocation problem of CC, RB, and power. In model $P_{1}$, the average MOS value of the user is employed as the weight of the resource allocation problem to maximize the total rate of all users by satisfying the carrier aggregation capabilities of different users and considering user fairness.

Because the optimal model $P_{1}$ is a nonlinear mixed optimization problem, it is divided into two subproblems: the $\mathrm{CC}$ and $\mathrm{RB}$ allocation problem $P_{11}$ and the power allocation problem $P_{12} . P_{11}$ is the allocation problem of CC and RB when the power allocation is known, and it is NP-hard. It is optimized by using an improved DABC algorithm called the DABC-CCRBA algorithm. This algorithm has the advantages of low computational complexity and fast convergence compared with existing CC and RB allocation algorithms. $P_{122}$ is a concave programming problem, and the SPA power allocation algorithm is proposed to solve it. In the SPA algorithm, the Lagrangian dual method is used to solve the problem of power allocation, where the Lagrangian dual variable is updated by the subgradient method. 
By using the above, the resource allocation algorithm JRA-WQOE was proposed. It is a cross-layer resource allocation algorithm that combines CC, RB, and power allocation and considers user QoE. In JRA-WQOE, $P_{1}$ is divided into two sub-problems $P_{11}$ and $P_{12}$. These subproblems are iterated until the algorithm reaches the termination condition. Simulation results showed that the proposed JRA-WQOE has the advantages of fast convergence and low computational complexity. Compared with the DABC-CCRBA+EPA algorithm, JCRPA algorithm, and Cross-CC-PF+EPA algorithm, the proposed JRA-WQOE enhanced the average user throughput and fairness. Under the condition of different numbers of users and SNRs, the JRA-WQOE algorithm also obtained the highest average user QoE value. Therefore, it further improves the average user throughput, fairness, and user satisfaction.

\section{References}

[1] Lim C., Yoo T., Clerckx B., Lee B. and Shim B., "Recent trend of multiuser mimo in lte-advanced," IEEE Communications Magazine, vol. 51, no. 3, pp. 127-135, March , 2013.

Article (CrossRef Link)

[2] Wang, Y., Pedersen, K. I., Rensen, T. B. and Mogensen, P. E., "Carrier load balancing and packet scheduling for multi-carrier systems,” IEEE Transactions on Wireless Communications, vol. 9, no. 5, pp. 1780-1789, May,2010. Article (CrossRef Link)

[3] Tian H., Gao S., Zhu J. and Chen L, "Improved Component Carrier Selection Method for Non-Continuous Carrier Aggregation in LTE-Advanced Systems," in Proc. of IEEE Vehicular Technology Conference, pp.1-5, September 5-8, 2011. Article (CrossRef Link)

[4] Jongheon Lee and Jaewoo So, "Analysis of Cognitive Radio Networks with Channel Aggregation," in proc. of 2010 Wireless Communications and Networking Conference (WCNC), pp.1-6, April 18-21, 2010. Article (CrossRef Link)

[5] Yuanye Wang, Klaus I. Pedersen, Preben E. Mogensen and Troels B. Sørensen, "Resource allocation considerations for multi-carrier LTE-Advanced systems operating in backward compatible mode," in Proc. of 2009 IEEE $20^{\text {th }}$ International Symposium on, pp.370-374, Sept. 13-16, 2009. Article (CrossRef Link)

[6] Yao-Liang Chung, Lih-Jong Jang and Zsehong Tsai, “An efficient downlink packet scheduling algorithm in LTE-Advanced systems with carrier aggregation,” in proc. of 2011 Consumer Communications and Networking Conference (CCNC), pp. 632-636, Jan. 9-12, 2011.

Article (CrossRef Link)

[7] Li Chen, Wenwen Chen, Xin Zhang and Dacheng Yang, “Analysis and simulation for spectrum aggregation in LTE-Advanced system,” in proc. of 2009 IEEE 70th Vehicular Technology Conference Fall, pp.1-6, Sept. 20-23, 2009. Article (CrossRef Link)

[8] Yuanye Wang, Klaus I. Pedersen, Troels B. Sorensen and Preben E. Mogensen, "Utility maximization in LTE-Advanced systems with carrier aggregation,” in proc. of 2011 IEEE 73rd Vehicular Technology Conference (VTC Spring) , pp.1-5, May15-18, 2011.

Article (CrossRef Link)

[9] L. Zhang, K. Zheng, W. Wang and L. Huang, "Performance analysis on carrier scheduling schemes in the long-term evolution-advanced system with carrier aggregation,” IET Communications, vol. 5, no.5, pp. 612-619, April, 2011. Article (CrossRef Link)

[10] Ji-hong Zhao, Hui Li and Qu Hua, "A SPF-PF crossing component carrier joint scheduling algorithm," in Proc. of 2012 14th International Conference on Advanced Communication Technology (ICACT), pp. 173-177, Feb. 19-22, 2012. Article (CrossRef Link)

[11] Fan Wu, Yuming Mao, Supeng Leng and Xiaoyan Huang, “A carrier aggregation based resource allocation scheme for pervasive wireless networks," in proc. of 2011 IEEE Ninth International Conference on Dependable, Autonomic and Secure Computing, pp. 196-201, Dec.12-14, 2011. Article (CrossRef Link) 
[12] Alexandru Vulpe, Octavian Fratu, Albena Mihovska and Ramjee Prasad, “A multi-carrier scheduling algorithm for LTE-Advanced," in proc. of 2013 16th International Symposium on Wireless Personal Multimedia Communications (WPMC) , pp. 1-5, June 24-27, 2013.

Article (CrossRef Link)

[13] Zhu Guohui, Zhou Guangtan and Xue Qian, "Wireless resource allocation algorithm for LTE-Advanced system with carrier aggregation," Journal of Xi'an University of Posts and Telecommunications, vol. 20, no. 2, pp. 47-51, Feb., 2015. Article (CrossRef Link)

[14] Ma Zheming, Wu Xuanli and Han Xingling, "Resource utilization based delay prioritized scheduler algorithm in LTE-A system,” Journal of Harbin Institute of Technology, vol. 48, no. 5, pp. 57-61, May, 2016. Article (CrossRef Link)

[15] Christos Tsinos, Apostolos Galanopoulos and Fotis Foukalas, "Low-Complexity and Low-Feedback-Rate Channel Allocation in CA MIMO Systems with Heterogeneous Channel Feedback,” IEEE Transactions on Vehicular Technology, vol. 66, no.5, pp. 4396-4409, May, 2017. Article (CrossRef Link)

[16] Christos G. Tsinos, Fotis Foukalas and Theodoros A. Tsiftsis, "Resource Allocation for Licensed/Unlicensed Carrier Aggregation MIMO Systems,” IEEE Transactions on Communications, vol. 65, no. 9, pp. 3765-3779, Sept., 2017. Article (CrossRef Link)

[17] Yuan Pu, Yong Xiao, Guoan Bi and Liren Zhang, “Toward Cooperation by Carrier Aggregation in Heterogeneous Networks: A Hierarchical Game Approach,” IEEE Transactions on Vehicular Technology, vol. 66, no. 2, pp. 1670-1683, Feb., 2017.Article (CrossRef Link)

[18] Wu F, Leng SP, Mao YM and Huang XY, “ A joint resource allocation scheme for OFDMA-based wireless pervasive networks with carrier aggregation,” International journal of Ad Hoc and ubiquitous computing, vol. 13, no. 2, pp. 109-119, Feb., 2013.Article (CrossRef Link)

[19] Zanjie Huang, Yusheng Ji, Hao Zhou and Baohua Zhao, "Joint Resource Allocation Algorithm in Carrier Aggregation Enabled Future Wireless Networks,” IEICE Transcactions on Fundamentals of Electrionics Communications and Computer Sciences, vol. E97.A , no. 1, pp. 78-85, Jan., 2014. Article (CrossRef Link)

[20] Yan P., Chen X., Li Z., and Jia Y., "QoS-Aware and Fair Resource Allocation with Carrier Aggregation in LTE-A Networks,” in Proc. of $201612^{\text {th }}$.International Conference on Mobile Ad-Hoc and Sensor Networks, pp. 97-104, Dec.,2016. Article (CrossRef Link)

[21] Shajaiah Haya, Abdelhadi Ahmed and Clancy T. Charles, "Towards an Application-Aware Resource Scheduling With Carrier Aggregation in Cellular Systems,” IEEE Communications Letters, vol. 20, no. 1, pp. 129-132, Jan., 2016. Article (CrossRef Link)

[22] Ali Ahmed A., Nordin Rosdiadee, Ismail Mahamod, ET AL.. Ali AA, Nordin R, Ismail M and Abdullah H, "An Efficient Scheduling Scheme for OFDMA Resource Blocks with Joint User Scheduling based on Earliest Deadline First with Carrier Aggregation (CA) in LTE-A System,” Wireless Personal Communications, vol. 88, no. 2, pp. 173-183, May, 2016. Article (CrossRef Link)

[23] Miha Rugelj, Urban Sedlar, Mojca Volk, Janez Sterle, Melita Hajdinjak and Andrej Kos, "Novel crosslayer QOE-Aware radio resource allocation algorithms in multiuser OFDMA systems," IEEE Transactions on Communications, vol. 62, no. 9, pp. 3196-3208, Sept., 2014. Article (CrossRef Link)

[24] Ducheng Wu, Qihui Wu, Yuhua Xu, Jianjun Jing and Zhiqiang Qin, “QoE-Based Distributed Multichannel Allocation in 5G Heterogeneous Cellular Networks: A Matching-Coalitional Game Solution,” IEEE Access, vol. 5, pp. 61-71, Feb., 2017.Article (CrossRef Link)

[25] Jiajia Liu, Shangwei Zhang, Nei Kato, Hirotaka Ujikawa, and Kenichi Suzuki, “Device-to-device communications for enhancing quality of experience in software defined multi-tier LTE-A networks,” Network IEEE, vol. 29, no. 4, pp. 46-52, Aug., 2015. Article (CrossRef Link)

[26] ITU, “Definition of Quality of Experience(QOE)”. Reference: TD 109 rev2(PLEN/12), ITU, Jan, 2007.

[27] R. Jain, “Quality of Experience,” IEEE Computer Socity Magazine, vol.11, no.1, pp.95-95, Jan., 2004. Article (CrossRef Link) 
[28] ITU-T Recommendation P.800. "Methods for Subjective Determination of Transmission Quality," 1996. Article (CrossRef Link)

[29] Lin Chuang, Hu Jie and Kong Xiangzhen, "Survey on models and evaluation of quality of Experience,” Chinese Journal of Computers, vol.35, no. 1, pp.1-15, Jan., 2012. Article (CrossRef Link)

[30] Andreas Saul and Gunther Auer, "Multiuser resource allocation maximizing the perceived quality," EURASIP Journal on wireless communications and networking, no.1, pp.1-15, Jan., 2009.

Article (CrossRef Link)

[31] Ameigeiras P, Ramos-Munoz JJ, Navarro-Ortiz J, Mogensen P and Lopez-Soler JM, "QOE oriented cross-layer design of a resource allocation algorithm in beyond 3G systems," Computer Communications, vol. 33, no .5, pp. 571-582, Mar, 2010. Article (CrossRef Link)

[32] Z.H. Ding, M. Huang and Z. R. Lu, "Structural damage detection using artificial bee colony algorithm with hybrid search strategy," Swarm and Evolutionary Computation, vol. 28, pp.1-13, June, 2016. Article (CrossRef Link)

[33] Shams K. Nseef, Salwani Abdullah, Ayad Turky and Graham Kendall, "An adaptive multi-population artificial bee colony algorithm for dynamic optimisation problems," Knowledge-Based Systems, vol.104, pp.14-23, July, 2016. Article (CrossRef Link)

[34] Ning Ai-ping and Zhang Xue-ying, "Convergence analysis of artificial bee colony algorithm," Control and Decision, vol. 28, no. 10, pp.1554-1558, Oct., 2013. Article (CrossRef Link)

[35] Wang Sheng-sheng, Yang Juan-juan and Chai Sheng, "Artificial Bee Colony Algorithm with Chaotic Catfish Effect and Its Application,” Acta Electronica Sinica, vol. 42, no. 9, pp. 1731-1737, Sept., 2014. Article (CrossRef Link)

[36] Zhao Hui, Li Mudong and Weng Xing-wei, "Improved artificial bee colony algorithm with self-adaptive global-guided quick searching strategy," Control and Decision, vol. 29, no. 11, pp. 2041-2047, Nov., 2014. Article (CrossRef Link)

[37] Yannis Marinakis, Magdalene Marinaki and Nikolaos Matsatsinis, " A hybird discrete artifical bee colony -GRASP algorithm for clustering," in Proc. of 2009 International Conference on Computers \& Industrial Engineering, pp. 548-553, July 6-9, 2009. Article (CrossRef Link)

[38] Zhu binglian, Zhu Fangfang, Duan Qingyan, Zhang Liang and Xiao Xinting, "An Improved Spectrum Allocation Algorithm Using Multi-Strategy Discrete Artificial Bee Colony Technology," Journal of Xi'an Jiaotong University, vol. 50, no. 2, pp. 20-25, Feb., 2016. Article (CrossRef Link)

[39] Abdellah Rezoug, Mohamed Bader-El-Den and Dalila Boughaci, "Knowledge-based Genetic Algorithm for the 0-1 Multidimensional Knapsack Problem," Evolutionary Computation 2017 IEEE congress on, pp. 2030-2037, June, 2017. Article (CrossRef Link)

[40] Tong Zhao, Li Bingbing and Hui Yongtao, "Spectrum allocation algorithm using improved particle swarm optimization," Journal of Huazhong University of Science and Technology(Natural Science Edition), vol. 41, no. 7, pp. 52-56, July, 2013. Article (CrossRef Link)

[41] Wei Yu and R. Lui, "Dual methods for nonconvex spectrum optimization of multicarrier systems," IEEE Transactions on Communications, vol. 54, no. 7, pp.1310-1322, July, 2006.

Article (CrossRef Link) 


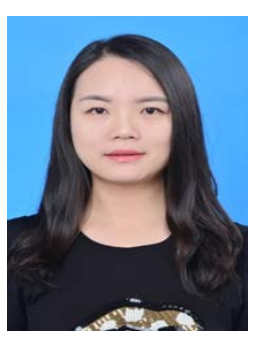

LIU Jun-Xia was born in 1980, is associate professor of Xinjiang Institute of Engineering of china. She received B.S. degree from Xinjiang Normal University of China. M.S. degree was obtained from Xinjiang University of China. She is a Ph. D. candidate of xinjaing university now. Her research interests are mobile communication network resource optimization, intelligence algorithm etc.

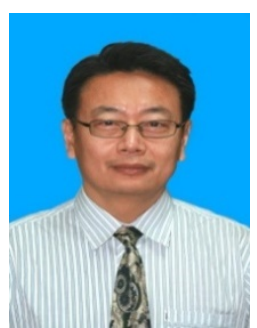

JIA Zhen-Hong was born in 1964, is doctoral supervisor and professor of Xinjiang University of China. He received B.S. degree from Beijing Normal University of China. M.S. degree and doctor's degree were obtained from Shanghai Jiao Tong University of China. At present, he is a member of the Optical Society of China holography and optical information processing Committee, Committee members of National optoelectronic technology and systems, doctoral supervisor of Xi'an Jiao Tong University and Xinjiang University of China. His research interests are optical communication technology, signal and information processing etc. 